\title{
Spectral broadening enhancement in silicon waveguides through pulse shaping
}

\author{
David Castelló-Lurbe, ${ }^{1, *}$ Enrique Silvestre, ${ }^{1}$ Pedro Andrés, ${ }^{1}$ and Víctor Torres-Company ${ }^{2,3}$ \\ ${ }^{1}$ Departament d'Òptica, Universitat de València, 46100 Burjassot, Spain \\ ${ }^{2}$ School of Electrical and Computer Engineering, Purdue University, West Lafayette, Indiana 47907, USA \\ ${ }^{3}$ Departament de Física, Universitat Jaume I, 12071 Castelló, Spain \\ *Corresponding author: david.castello-lurbe@uv.es
}

Received February 6, 2012; revised May 2, 2012; accepted May 4, 2012;

posted May 11, 2012 (Doc. ID 162636); published June 29, 2012

\begin{abstract}
Spectral broadening in silicon waveguides is usually inhibited at telecom wavelengths due to some adverse effects related to semiconductor dynamics, namely, two-photon and free-carrier absorption (FCA). In this Letter, our numerical simulations show that it is possible to achieve a significant enhancement in spectral broadening when we properly preshape the input pulse to reduce the impact of FCA on spectral broadening. Our analysis suggests that the use of input pulses with the correct skewness and power level is crucial for this achievement. (c) 2012 Optical Society of America
\end{abstract}

OCIS codes: $130.4310,190.4360,190.4390$.

Nowadays, continuous advances in nanophotonics technologies have spurred on interest in the study of silicon-based optical materials [1]. Strong confinement of light in nanoengineered silicon-on-insulator (SOI) waveguides results in a huge effective nonlinearity and the ability for dispersion engineering (see, e.g., [2,3]). These achievements have opened up the possibility of performing previously demonstrated signal processing functionalities (mainly based in nonlinear kilometric fibers) at chip scale with relatively low optical power [4]. These Si-based components offer the benefits of low cost (complementary metal-oxide-semiconductorcompatible large-scale-fabrication processes) and low power consumption.

However, at the relevant wavelength region around $1.5 \mu \mathrm{m}$, Kerr-based spectral broadening or self-phase modulation (SPM) is accompanied by an orchestra of different nonlinear phenomena arising from the semiconductor carrier dynamics [므느. Specifically we mention the absorption and dispersion of free carriers produced by two-photon absorption (TPA), which are not present in conventional silica-based devices. The net effect results in a depletion of the achievable spectral broadening for a Gaussian input pulse in comparison with the case when only SPM is acting [ $\underline{8}-\underline{10}]$.

Additionally, the phenomenon under study is extremely sensitive to the input pulse characteristics due to the inherent nonlinear nature of the spectral broadening. In fact, up to some extent, pulse shaping techniques have demonstrated to be effective in controlling the nonlinear broadening in photonic crystal fibers $[11,12]$ and other nonlinear materials [13-15], using both single-pass [15] and self-learning adaptive configurations [11-14]. Besides, these techniques offer valuable insight in understanding the pulse dynamics through propagation $[11,16]$. In this Letter, we show that the proper manipulation of the pulse phase enhances spectral broadening even in the presence of TPA and free-carrier absorption (FCA).

Let us remind the reader that the dynamics of an optical pulse propagating in an SOI nanowaveguide can be described in mathematical terms by [9]

$$
\begin{gathered}
\partial_{z} A+i \frac{\beta_{2}}{2} \partial_{t}^{2} A=i \gamma_{0}(1+i r)|A|^{2} A-\frac{\sigma}{2}(1+i \mu) N_{c} A-\frac{\alpha_{l}}{2} A, \\
N_{c}(z, t)=\frac{\beta_{\mathrm{TPA}}}{2 h \nu_{0} A_{\mathrm{eff}}^{2}} \int_{-\infty}^{t} e^{-\frac{t-t^{\prime}}{\tau_{c}}}\left|A\left(z, t^{\prime}\right)\right|^{4} \mathrm{~d} t^{\prime},
\end{gathered}
$$

where $A$ is the electric-field envelope, $\nu_{0}$ represents the carrier frequency, $\beta_{2}$ stands for the group velocity dispersion (GVD) parameter, $n_{2}$ is the Kerr coefficient, $A_{\text {eff }}$ is the effective area of the waveguide, $\gamma_{0}$ denotes the nonlinear coefficient of the waveguide defined as $\gamma_{0}=n_{2} 2 \pi \nu_{0} / c A_{\text {eff }}, \sigma$ is the FCA coefficient, $\alpha_{l}$ the linear loss coefficient, $N_{c}$ corresponds to the carrier density, $\beta_{\text {TPA }}$ is the TPA coefficient, $\tau_{c}$ represents the carrier lifetime, and the dimensionless parameters $r=\beta_{\mathrm{TPA}} c /$ $4 \pi n_{2} \nu_{0}$ and $\mu$ account for the relative weight of TPA and free-carrier dispersion (FCD), respectively. As in previously published results [9], we consider $\lambda_{0}=1.55 \mu \mathrm{m}$, $\alpha_{l}$ corresponding to $1 \overline{\mathrm{dB}} / \mathrm{cm}, \quad n_{2} \approx 6 \times 10^{-18} \mathrm{~m}^{2} / \mathrm{W}$, $\beta_{\mathrm{TPA}}=5 \times 10^{-12} \mathrm{~m} / \mathrm{W}, \quad \sigma=1.45 \times 10^{-21} \mathrm{~m}^{2}, \quad r=0.1$, $\mu=7.5$, and $\tau_{c}=1 \mathrm{~ns}$. Likewise, in our numerical simulations we choose $A_{\text {eff }} \approx 1 \mu \mathrm{m}^{2}$. Because we focus our attention on the interplay between SPM and carrier effects, we consider quite long pulses for which the GVD term in Eq. (1) shall be neglected in the following.

In order to analyze in a qualitative way the spectral broadening, it is very useful to study the instantaneous frequency of the propagated complex field. With this aim, let us explicitly consider the phase of the complex field envelope, $A=|A| \exp (i \phi)$. By defining the effective $z$-dependent loss coefficient and length, $\alpha_{\mathrm{eff}}=\alpha_{l}+\sigma N_{c}$ and $z_{\text {eff }}=\int_{0}^{z} \exp \left(-\int_{0}^{z^{\prime}} \alpha_{\mathrm{eff}} \mathrm{d} z^{\prime \prime}\right) \mathrm{d} z^{\prime}$, respectively, the following key equations are derived in an analogous way to Eq. (60) in [ㅁ]:

$$
\partial_{z}\left(-\partial_{t} \phi\right)=-\gamma_{0} \partial_{t}|A|^{2}+\frac{\sigma \mu}{2}\left(\frac{\beta_{\mathrm{TPA}}}{2 h \nu_{0} A_{\mathrm{eff}}^{2}}|A|^{4}-\frac{N_{c}}{\tau_{c}}\right),
$$




$$
|A|^{2}=\frac{\exp \left(-\int_{0}^{z} \alpha_{\mathrm{eff}} \mathrm{d} z^{\prime}\right) A_{0}^{2}}{1+2 \gamma_{0} r z_{\mathrm{eff}} A_{0}^{2}},
$$

where $A_{0}=|A(0, t)|$.

Despite their complexity, it is possible to get some valuable physical insight from the above equations. From Eq. (3), we note that the evolution of the instantaneous frequency is governed by two processes. The first term of the right-hand side corresponds to SPM, whereas the second one takes into account FCD. For low repetition-rate pulses and if the input pulse width $T_{0} \ll \tau_{c}$, the subterm proportional to $-N_{c} / \tau_{c}$ can be neglected [9], and hence the FCD term can be considered always positive. Accordingly, FCD only produces blueshifted components; by contrast SPM can give rise to both blueshifted and redshifted ones $[\underline{9}, \underline{10}]$. Finally, similarly to [ㅎ], we define the threshold power $P_{\text {th }}=8 \gamma_{0} h \nu_{0} A_{\text {eff }}^{2} / T_{0} \sigma \mu \beta_{\mathrm{TPA}}$, beyond which the FCD contribution exceeds that of SPM.

Having in mind the above facts, we realize that an asymmetric input pulse induces two concomitant effects. On one hand, the longer wavelength production due to SPM is improved by means of a skew input pulse with positive sign due to the enhanced slope on the leading edge of the pulse. On the other hand, if the peak power $P_{0} \gg P_{\text {th }}$, the shorter wavelengths are generated earlier in time, near to the peak power. According to Eq. (2), the production of free carriers is a cumulative effect, and hence, as one can see in Eq. (4), FCA highly affects the trailing edge of the pulse. So, losses induced by FCA could be partially compensated by generating blueshifted frequencies so fast that the free carriers did not have time to accumulate. Moreover, a positive skewness of the pulse profile would strengthen this beneficial effect. Thus, both longer and shorter wavelength enhancement could be feasible using positive skew pulses.

To prove the above ideas, we solve the previous equations considering two kinds of skew input pulses produced by adding a certain spectral phase to a Gaussian pulse (hence preserving its bandwidth and energy). Both cases could be experimentally implemented in a feasible way by means of a pulse shaper with good spectral resolution. First we consider a spectral phase of the form $\eta\left(\omega-\omega_{0}\right)^{3}$, where $\eta$ denotes the strength of the skewness. The negative $\eta$ sign guarantees the right skewness of the temporal pulse profile.

We have solved numerically Eqs. (1) and (2) and computed the achieved spectral width at $-20 \mathrm{~dB}$ for different values of the $\eta$ parameter. In this calculation, we consider $T_{0}=10 \mathrm{ps}$ and a $1 \mathrm{~cm}$ long silicon waveguide. The results are shown in Fig. 1 and support our explanation. The maximum broadening is achieved at $\eta=-125 \mathrm{ps}^{3}$ for $100 \mathrm{~W}$ and $\eta=-124 \mathrm{ps}^{3}$ for $1 \mathrm{~kW}$ (peak powers are referred to the unmodulated Gaussian pulse in both cases, unshaped and shaped). Only the SPM effect is working at $10 \mathrm{~W}$, and, therefore, no significant improvement of the spectral broadening is observed. Note that, in our numerical example, $P_{\text {th }}$ is about $50 \mathrm{~W}$. When we increase the input power up to $100 \mathrm{~W}$ [see Fig. 2(a)], the SPM production of low frequencies is intensified by the pulse asymmetry. In addition, the upper part of the spectrum remains unaffected. Here the FCA changes induced

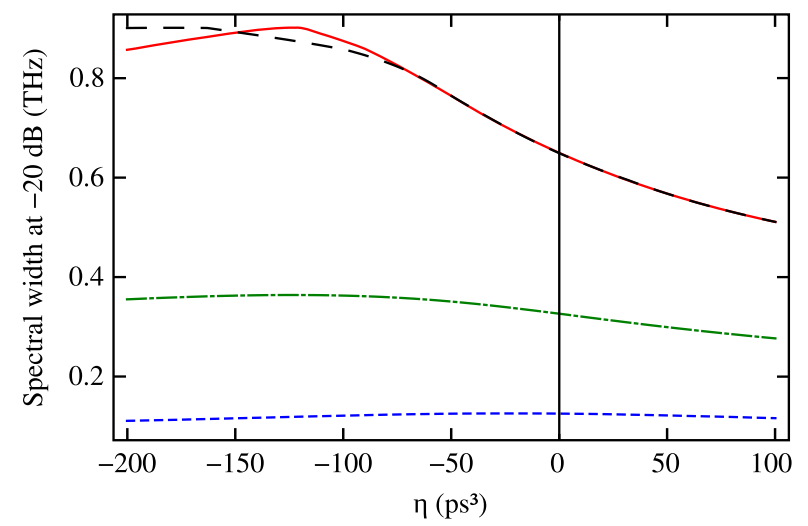

Fig. 1. (Color online) Spectral output width at $-20 \mathrm{~dB}$ as a function of the strength $\eta$ of the cubic-phase modulation for three input peak powers: $10 \mathrm{~W}$ (short-dashed curve), $100 \mathrm{~W}$ (dotteddashed curve), and $1 \mathrm{~kW}$ (solid curve). The long-dashed curve corresponds to the input spectral phase provided by the Gerchberg-Saxton (GS) algorithm (see details in the text).

by pulse shaping do not provide an enhancement of higher frequencies because of the reduction on blueshifted components produced by SPM due to the smooth tail of the asymmetric pulse. At higher (but realistic; see [10]) powers, a notable enhancement appears at both sides of the spectrum since FCA plays a less significant role thanks to the pulse shape. The expected output spectrum is depicted in Fig. 2(b). In this case, it is even up to $40 \%$ broader with respect to the unshaped case (measured at the $-20 \mathrm{~dB}$ level).

Finally, we stress the connection between reducing FCA and spectral broadening by computing $N_{c}$ at the instant when the maximum of the output pulse occurs; see Fig. 3(a). It is worth noting that the spectral width reduction shown by the curve corresponding to $P_{0}=1 \mathrm{~kW}$ in
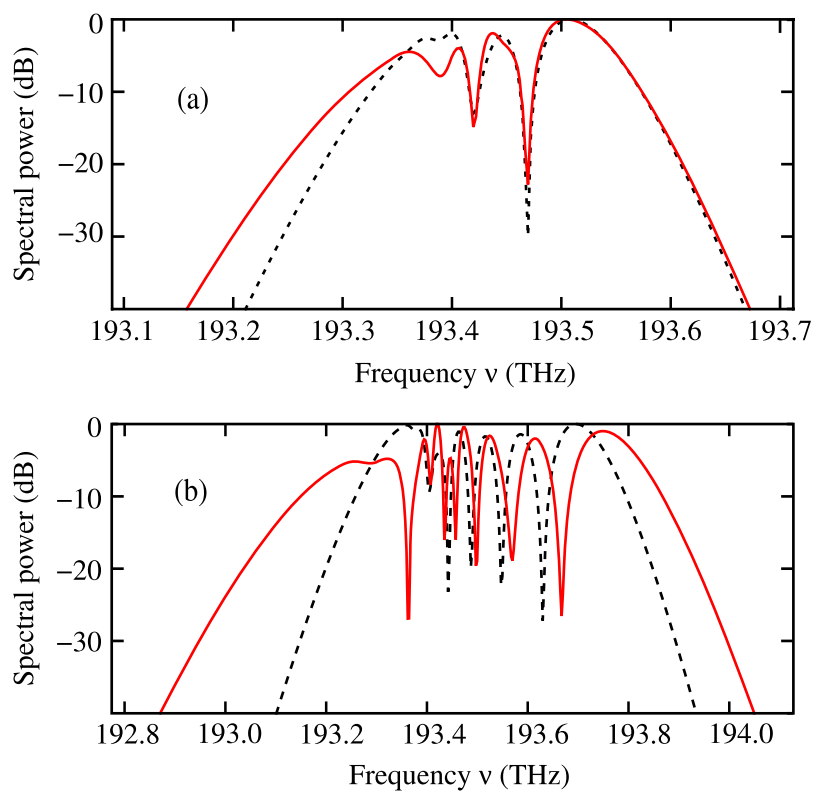

Fig. 2. (Color online) Comparison of the output power spectrum between the unmodulated Gaussian input pulse (dashed curve) and the spectrally chirped version (solid curve) for (a) $P_{0}=100 \mathrm{~W}, \eta=-125 \mathrm{ps}^{3}$ and (b) $P_{0}=1 \mathrm{~kW}$, $\eta=-124 \mathrm{ps}^{3}$. 

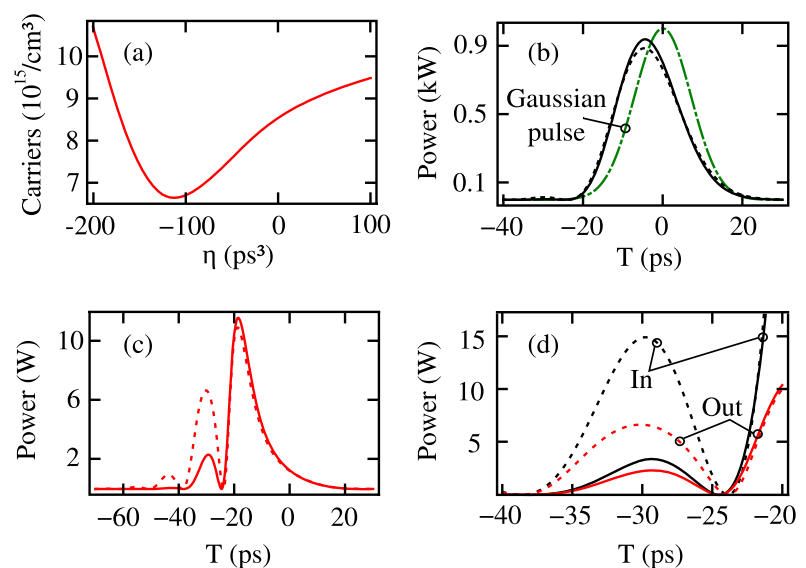

Fig. 3. (Color online) (a) Density of carriers at the maximum of the output pulse in terms of $\eta$; (b) input pulses; (c) output pulses. In both plots (b) and (c), dashed curves correspond to pulses resulting from the cubic-phase modulation and solid curves refer to pulses resulting from the GS algorithm. In all cases, $\eta=-200 \mathrm{ps}^{3}$ and $P_{0}=1 \mathrm{~kW}$. (d) Secondary lobes of the modulated pulses of (b) and (c).

Fig. 1 is consistent with the increase of $N_{c}$ for lower values of $\eta$. Moreover, we link this feature with the oscillatory structure that appears near the leading edge of the input pulse due to the cubic spectral phase used to obtain skew input pulses in these cases; see Figs. 3(b)-3(d). These secondary lobes generate additional carriers that enhance FCA where the production of new frequencies is concentrated.

Note that, for greater values of positive skewness, the pulse shows bigger ripples before the main lobe. In order to confirm this assumption, with the aid of the GS algorithm [17], we search for spectral phases that preserve the shape of the main lobe of the pulses shaped through a cubic spectral phase. Accordingly, for the different values of the $\eta$ parameter considered previously, we take $\eta\left(\omega-\omega_{0}\right)^{3}$ as our seed phase (this choice accelerates the convergence of the algorithm) and set our target as the main lobe of the corresponding $\eta$-skew pulse (i.e., the above referred oscillations of the preshaped pulses are eliminated to construct the targets). Every GS solution is labeled with the $\eta$ value of the corresponding seed phase. According to Figs. 3(c) and 3(d), the GS technique provides a reduction of the satellite lobes but not their total suppression. The resulting spectral behavior shown in Fig. 1 confirms the pernicious effect of the secondary lobes when input pulses present sufficient skewness.

In summary, we have shown that the skewness introduced on a Gaussian input pulse of high enough peak power leads to an spectral broadening enhancement on SOI waveguides. The key point is to enhance longer wavelength production due to SPM by increasing the leading slope edge which simultaneously decreases FCA effects on shorter wavelength production. Although in this work we do not take into account dispersive phenomena, our physical interpretation suggests that the current achievements can also be extended to this case.

This work was financially supported by the Plan Nacional Investigación, Desarrollo e Innovación (I+D + I) under the research project TEC2008-05490, by Ministerio de Ciencia e Innovación (Spain), and by the Generalitat Valenciana under the grant PROMETEO 2009-077. One of the authors, D. C.-L., gratefully acknowledges funding from the Generalitat Valenciana (VALi+d predoctoral contract). V. T.-C. gratefully acknowledges funding from a Marie Curie International Outgoing Fellowship (project PIOF-2009-234996).

\section{References}

1. B. Jalali and S. Fathpour, J. Lightwave Technol. 24, 4600 (2006).

2. A. C. Turner-Foster, M. A. Foster, R. Salem, A. L. Gaeta, and M. Lipson, Opt. Express 18, 1904 (2010).

3. L. Zhang, Y. Yue, R. G. Beausoleil, and A. E. Willner, Opt. Express 19, 8102 (2011).

4. J. Leuthold, C. Koos, and W. Freude, Nat. Photon. 4, 535 (2010).

5. Ö. Boyraz, P. Koonath, V. Raghunathan, and B. Jalali, Opt. Express 12, 4094 (2004).

6. Q. Lin, O. J. Painter, and G. P. Agrawal, Opt. Express 15, 16604 (2007).

7. R. M. Osgood, Jr., N. C. Panoiu, J. I. Dadap, X. Liu, X. Chen, I.-W Hsieh, E. Dulkeith, W. M. Green, and Y. A. Vlasov, Adv. Opt. Photon. 1, 162 (2009).

8. E. Dulkeith, Y. A. Vlasov, X. Cheng, N. C. Panoiu, and R. M. Osgood, Jr., Opt. Express 14, 5524 (2006).

9. L. Yin and G. P. Agrawal, Opt. Lett. 32, 2031 (2007).

10. P. Koonath, D. R. Solli, and B. Jalali, Appl. Phys. Lett. 93, 091114 (2008).

11. S. $\mathrm{Xu}, \mathrm{D}$. H. Reitze, and R. S. Windeler, Opt. Express 12, 4731 (2004).

12. D. Lorenc, D. Velic, A. N. Markevitch, and R. J. Levis, Opt. Commun. 276, 288 (2007).

13. F. G. Omenetto, A. J. Taylor, M. D. Moores, and D. H. Reitze, Opt. Lett. 26, 938 (2001).

14. D. Schumacher, Opt. Lett. 27, 451 (2002).

15. X. Yang, D. J. Richardson, and P. Petropoulos, in CLEO/ Europe and EQEC 2011 Conference Digest, OSA Technical Digest (CD) (Optical Society of America, 2011), CI2_1.

16. C. Ament, P. Polynkin, and J. V. Moloney, Phys. Rev. Lett. 107, 243901 (2011).

17. A. Rundquist, A. Efimov, and D. H. Reitze, J. Opt. Soc. Am. B 19, 2468 (2002). 